\title{
Varying coefficient analysis for indeterminate species interactions with non-parametric estimation, exemplifying with a fig-fig wasp system
}

\author{
SHI Lei ${ }^{1,2 \dagger}$, WANG RuiWu ${ }^{2 \dagger *}$, ZHU LiXing $^{3 \dagger}$, ZEN WeiMing $^{4}$, XU WangLi $^{5} \&$ ZHENG Qi $^{2}$ \\ ${ }^{1}$ Statistics and Mathematics School, Yunnan University of Finance and Economics, Kunming 650221, China; \\ ${ }^{2}$ Ecology, Conservation, and Environment Center (ECEC), State Key Laboratory of Genetic Resources and Evolution, Kunming Institute of Zoo- \\ logy, Chinese Academy of Sciences, Kunming 650223, China; \\ ${ }^{3}$ Department of Mathematics, Hong Kong Baptist University, Hong Kong, China; \\ ${ }^{4}$ Scientific and Technical Department, Yunnan University of Nationalities, Kunming 650031, China; \\ ${ }^{5}$ Statistics College, RenMing University of China, Beijing 100080, China
}

Received February 25, 2011; accepted May 13, 2011

Research on species interactions has generally assumed that species have a fixed interaction and therefore linear or non-linear parametric regression models (e.g. exponential, logistic) have been widely used to describe the species interaction. However, these models that describe the relationship between interacting species as a specific functional response might not be appropriate for real biological communities, for instance, in a chaotic system, when the species relationship varies among different situations. To allow a more accurate description of the relationship, we developed a species correlation model with varying coefficient analysis, in which a non-parametric estimation is applied to identify, as a function of related factors, variation in the correlation coefficient. This was applied to a fig-fig wasp model system. When the effect of the factors on the relationship can be described with parameters, the new method reduces to traditional parametric correlation analysis. In this way, the new method is more general and flexible for empirical data analyses, but different by allowing investigation of whether a species interaction varies with respect to factors, and of the factors that maintain or change the species interaction. This method will have important applications in both theoretical and applied research (e.g. epidemiology, community management).

species interaction, non-parametric estimation, correlation coefficient, varying coefficient analysis, chaotic oscillations, density dependence, indeterminate interaction

Citation: Shi L, Wang R W, Zhu L X, et al. Varying coefficient analysis for indeterminate species interactions with non-parametric estimation, exemplifying with a fig-fig wasp system. Chinese Sci Bull, 2011, 56: 2545-2552, doi: 10.1007/s11434-011-4564-2

Study of species interactions is fundamental for understanding community ecology. Species are usually assumed to have a fixed interaction that can be described through correlation analysis with quantitative characteristics (e.g. fitness or population size, individual growth rate, immune or chemical response of individuals). Based on this assumption, linear regression or non-linear parametric regression that can transform to linear regression, which describe the

\footnotetext{
$\dagger$ These authors contributed equally to this work.

* Corresponding author (email: ruiwukiz@ @otmail.com)
}

effect of variables as a constant correlation coefficient, is commonly used to describe the species interaction [1,2]. In these analyses, the quantitative relationship of interacting species is expected to be determinate, namely it can be represented by a specific value [1,3-9]. Other factors are usually assumed to linearly affect biological characteristics of the involved species [7,10-13]. In statistical analyses, these variables are treated as covariates when a general linear model is applied [1,2,14]; however, this method is not appropriate for the correlation analysis of interacting variables in many biological systems, including species interaction 
analysis [15-18].

In a real ecological community, the relationship between interacting species might not be described by a constant correlation coefficient. For instance, the species relationship might be non-linear [16], and even chaotically oscillating, with the variation of other factors, which can be described as indeterminate interactions [13,19-22]. The species relationship might vary according to different environmental or ecological conditions, population densities of the involved species, or other factors $[3,4,20,22-27]$. In other words, any specific relationship may not be expected for such interacting species. This phenomenon has been reported in many ecological systems with inter-specific interactions ranging from mutualism to coexistence, to antagonism (e.g. predators and prey or parasites and hosts), [19,22,24,27-31]. Unfortunately, there are relatively few methods available in the literature that can quantitatively describe this phenomenon.

To address this problem, a natural approach is to fit the underlying relationship as a model with which we can investigate the correlation coefficients at different values of influential factors. This allows a more comprehensive analysis than that possible by traditional methods. To this end, we propose in this paper a new species interaction model using varying coefficient analysis and apply this to a fig wasp and its host system. This method for a general species interaction model and analysis includes the traditional method as a special case.

\section{A brief review of linear regression models}

Let $X$ and $Y$ denote the species quantitative characteristics (variables). If the species interaction can be represented by a constant value across quantitative characteristics, a linear regression model can describe their interaction as

$$
Y=\alpha_{0}+\alpha_{1} X+\varepsilon
$$

where the slope $\alpha_{1}$ is equivalent to the correlation coefficient between $Y$ and $X$, which is used to describe the interacting behavior of the two species. More general models such as the exponential or logistic model can also be transformed to be of the above structure when appropriate transformations on $X$ and $Y$ are performed. Thus, without loss of generality, we can focus on this model. If interacting species have a fixed or constant relationship, a significant positive slope of species quantitative characteristics will be expected for cooperative species, while a significant negative value will be expected for antagonistic species and a nonsignificant slope will be expected between non-interacting species or coexisting species that live in the same habitat but have different niches [3,5-8,11,12,32].

\subsection{Varying coefficients models}

Studies have shown that species interactions can vary according to temperature, season and other abiotic factors including resource availability $[22,27,33]$. Species interactions can also be conspecific density-dependent, such as through competition, or vary with the population density of other species $[16,34,35]$. This variation can cause large changes in the interaction between species [16]. The species interaction even changes from positive to negative in some well documented ecological systems, such as mutualism between the moth Greya politella and its host plant Lithophragmaparviflorum (Saxifragaceae), and mutualism between fig pollinator wasps and their host plant figs $[27,29,36]$, and the parasite-host system between the parasite Platyneura agraensis and its host Ceratosolen fusciceps [33].

Factors that might lead to variation of the correlation coefficient of species' quantitative characteristics can be described by variable $Z$, a regression model with functional coefficients that can be expressed as

$$
Y=\alpha_{0}(Z)+\alpha_{1}(Z) X+\varepsilon,
$$

where $\varepsilon$ is random error with a mean of zero that is independent of $Z, \alpha_{0}(Z)$ and $\alpha_{1}(Z)$ are the intercept and slope, respectively, both of which are functions of $Z$. In model (2), if $\alpha_{1}(Z)$ is independent of the third variable $Z$ (i.e. constant slope), then it reduces to the classical linear regression model (1). If the effect of $Z$ is not constant for species quantitative characteristics of the interacting species, this model is called a varying coefficient regression model [37]. Let $\rho_{X Y \mid Z}$ denote the conditional correlation coefficient of $X$ and $Y$ when $Z$ is given. This can be expressed as

$$
\alpha_{1}(Z)=\rho_{X Y \mid Z}\left(\sigma_{Y \mid Z} / \sigma_{X \mid Z}\right),
$$

where $\sigma_{X \mid Z}^{2}$ and $\sigma_{Y \mid Z}^{2}$ are the conditional variances of $X$ and $Y$ for a given $Z$ respectively. If $\sigma_{Y \mid Z} / \sigma_{X \mid Z}$ is not significantly dependent on $Z$, the correlation coefficient $\rho_{X Y \mid Z}$ as a function of $Z$ is equivalent to $\alpha_{1}(Z)$.

In model (2), the functional forms of $\alpha_{0}(Z)$ and $\alpha_{1}(Z)$ are not pre-specified, and a general method employing nonparametric smoothing technique can be used to estimate these functions. This nonparametric estimation method is advantageous because no functional forms need to be pre-specified, and it only uses information from data to obtain an estimated curve/function. Such a model is more flexible and robust than any parametric method [38], because there is a risk of making incorrect assumptions and conclusions with a parametric model of an assumed structure. Let $\alpha(Z)=\left(\alpha_{0}(Z), \alpha_{1}(Z)\right)^{\prime}, U=(1, X)^{\prime}$ where the prime stands for the transpose of the vector. When the matrix $E\left(U U^{\prime} \mid Z\right)$ is invertible, a least squares type estimator of $\alpha(Z)$ is uniquely defined by

$$
\hat{\alpha}(Z)=E\left(U U^{\prime} \mid Z\right)^{-1} E(U Y \mid Z) .
$$

This conditional expectation can be estimated by nonparametric smoothing methods such as kernel estimation [38]. Let $\left\{\left(X_{i}, Y_{i}, Z_{i}\right), i=1,2, \cdots, n\right\}$ be a sample of observa- 
tions. Then

$$
\begin{aligned}
& E\left(U U^{\prime} \mid Z\right)=\frac{1}{n} \sum_{i=1}^{n} w_{n i}(Z) U_{i} U_{i}^{\prime}, \\
& E\left(U Y^{\prime} \mid Z\right)=\frac{1}{n} \sum_{i=1}^{n} w_{n i}(Z) U_{i} Y_{i},
\end{aligned}
$$

where $U_{i}=\left(1, X_{i}\right)^{\prime}, w_{n i}(Z)=K_{h}\left(Z-Z_{i}\right) / f_{h}(Z), f_{h}(Z)=n^{-1} \sum_{i=1}^{n} K_{h}\left(Z-Z_{i}\right)$ and $K_{h}(u)=h^{-1} K(u / h)$. The kernel function $K(u)$ is a continuous, bounded and symmetric real function that integrates to one (e.f. $\int K(u) \mathrm{d} u=1$ ). A scalar $h$ is defined as the bandwidth. A variety of kernel functions are possible. Some commonly used kernel functions include the Epanechnikov kernel [38] with $K(u)=0.75\left(1-u^{2}\right) I(|u| \leqslant 1)$ and the quadratic kernel with $K(u)=\frac{15}{16}\left(1-u^{2}\right)^{2} I(|u| \leqslant 1)$ [39], where $I(|u| \leqslant 1)$ is an indicator function taking the value 1 when $|u| \leqslant 1$, and 0 otherwise. The weight function $w_{n i}(Z)$ is zero outside the interval $\left|Z-Z_{i}\right| \leqslant h$. It has been shown that large $h$ will lead to large bias and small variance of the estimator, which is called over-smoothing and small $h$ will lead to small bias and large variance, which is called undersmoothing. An appropriate bandwidth $h$ can be selected by the leave-one-out cross validation method [39]. In applications, one can subjectively choose the bandwidth $h$ to either over-smooth or under-smooth the curve based on the particular aim of the analysis [39].

Although we can identify from the fitted curves how the regression coefficients vary with $Z$, the functional forms of the curves are unknown. A method to test whether $\alpha_{1}(Z)$ is of a particular functional form can be performed using a Nonparametric Monte Carlo Test (NMCT) [40].

In the varying coefficient regression model, the correlation coefficient $\rho_{X Y \mid Z}$ can be well described by the regression coefficient $\alpha_{1}(Z)$ only when the ratio $\sigma_{Y \mid Z} / \sigma_{X \mid Z}$ is constant. However, when this condition is not satisfied, $\alpha_{1}(Z)$, as a function of $Z$, is not equivalent to $\rho_{X Y \mid Z}$ and a nonparametric estimation of $\rho_{X Y \mid Z}$ is desired. This can be seen from the definition

$$
\rho_{X Y \mid Z}=\frac{\operatorname{Cov}(X, Y \mid Z)}{\sqrt{\operatorname{Var}(X \mid Z) \operatorname{Var}(Y \mid Z)}}=\frac{\sigma_{X Y \mid Z}}{\sigma_{X \mid Z} \sigma_{Y \mid Z}} .
$$

The conditional covariance of $X$ and $Y$, and the variances of $X$ and of $Y$ when $Z$ is given can be computed by using kernel estimation [39]. For example, using the same notations, we have

$$
\begin{gathered}
\sigma_{X Y \mid Z}=\frac{1}{n} \sum_{i=1}^{n} w_{n i}(Z)\left(X_{i}-\hat{E}(X \mid Z)\right)\left(Y_{i}-E(Y \mid Z)\right), \\
\sigma_{X \mid Z}^{2}=\frac{1}{n} \sum_{i=1}^{n} w_{n i}(Z)\left(X_{i}-\hat{E}(X \mid Z)\right)^{2}
\end{gathered}
$$

$$
\sigma_{Y \mid Z}^{2}=\frac{1}{n} \sum_{i=1}^{n} w_{n i}(Z)\left(Y_{i}-\hat{E}(Y \mid Z)\right)^{2},
$$

where $\hat{E}(X \mid Z)=\frac{1}{n} \sum_{i=1}^{n} w_{n i} X_{i}$ and $\hat{E}(Y \mid Z)=\frac{1}{n} \sum_{i=1}^{n} w_{n i} Y_{i}$.

Even for such a complex structure, we can, similar to the linear model, still use the correlation coefficient $\left|\rho_{X Y \mid Z}\right|$ to reveal the correlation between variables although it is now a function of another factor.

\subsection{Simulation study}

The computation of the nonparametric estimation for the varying coefficient model proposed in this paper has been programmed using Matlab code. Estimation for the varying coefficients $\alpha_{1}(Z)(i=0,1)$ is written by a Matlab function called cvm.m, and the estimation for the correlation coefficient is contained in npcc.m. We conducted a simulation study to examine the performance of the new method for varying coefficient analysis.

In the simulations, we considered different forms of the functions $\alpha_{1}(Z)$, and set $\alpha_{0}(Z)$ to be a constant. We used constant, linear and other two special functions of $\alpha_{1}(Z)$ to simulate data drawn from the model $y=\alpha_{0}(Z)+\alpha_{1}(Z) x+\varepsilon$, where $Z$ is a random number from 0 to $8, x$ and $\varepsilon$ are standard normal random variables, and the sample size is 300 . For each $\alpha_{1}(Z)$, we obtained the generated dataset $\left\{\left(y_{i}, x_{i}\right.\right.$, $\left.\left.z_{i}\right), i=1, \ldots, n\right\}$, then calculated the estimated values of $\hat{\alpha}_{1}(z)$ using the method explained in the previous section, where the bandwidth $h$ in all plots was chosen as $h=0.5$ by an ad-hoc method. Plots of $y$ versus $x$ and $\hat{\alpha}_{1}(z)$ versus $z$ (together with $\alpha_{1}(Z)$ versus $z$ ) are shown in Figures 1 and 2. The results showed that when $\alpha_{1}(Z)$ is a constant, $y$ and $x$ are linearly related as shown in Figure 1(a), and the estimated $\hat{\alpha}_{1}(z)$ is also approximately a constant as shown in Figure 1(b). However, when $\alpha_{1}(Z)$ is not a constant function of $z$, for either linear or other specified forms, the plots of $y$ versus $x$, as shown in Figure 1(c), Figure 2(a) and (c), show that $y$ and $x$ no longer follow a linear relationship. However, our method still provides a good estimator $\hat{\alpha}_{1}(z)$ of $\alpha_{1}(Z)$, which sufficiently fits the given functions of $\alpha_{1}(Z)$, as shown in Figures 1(d), 2(b) and (d).

These simulation results suggest that when the regression coefficients are functions of other factors, the use of linear regression with constant coefficients is not appropriate, while the varying correlation coefficient analysis performs well to identify the underlying correlation patterns.

\section{Example of empirical data analysis}

We use a fig-fig wasp reciprocal mutualism interaction to illustrate our proposed analysis method. Fig-fig wasp mutualism is 

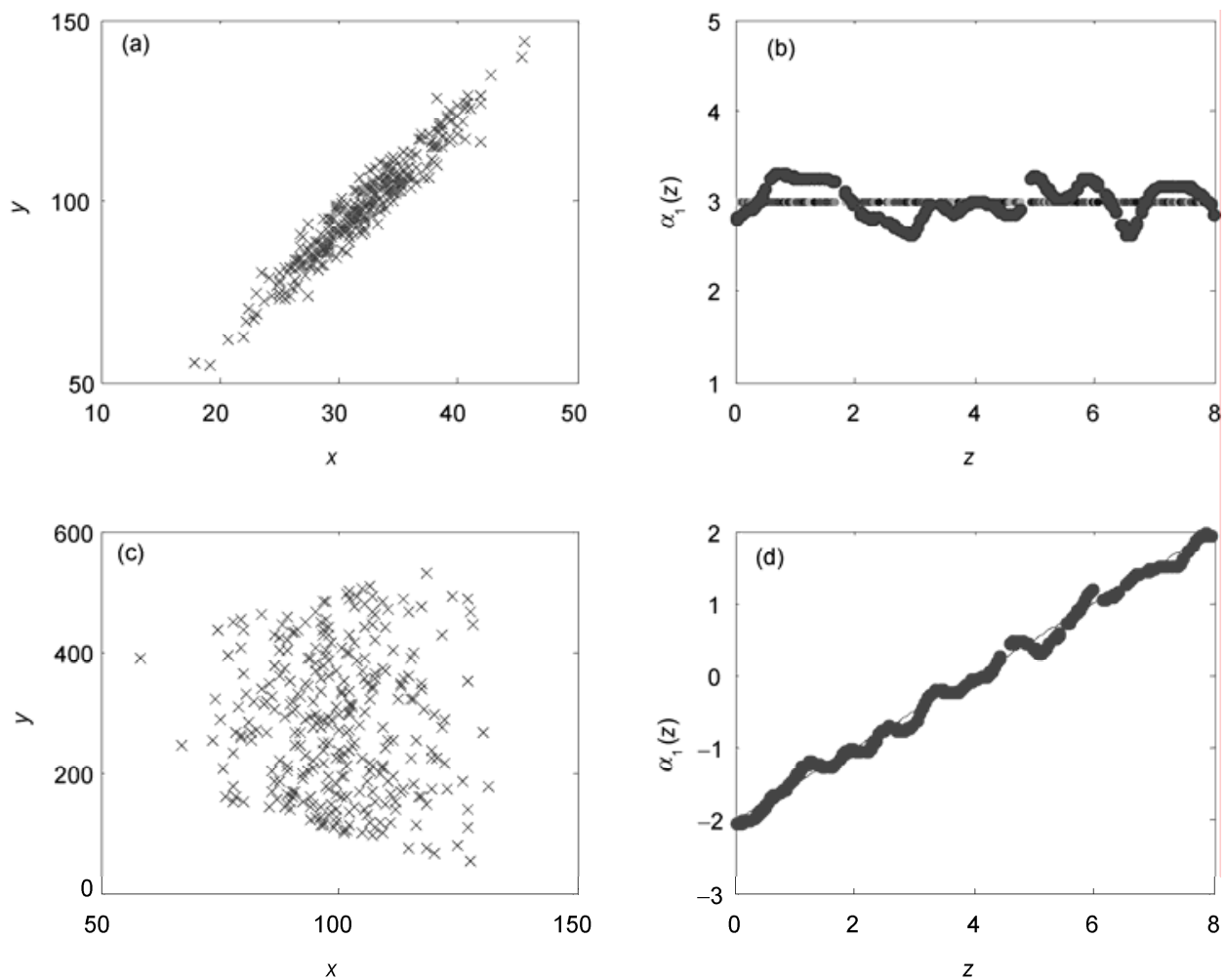

Figure 1 (a) Plot of $y$ versus $x$ for data simulated from the model $y=2+\alpha_{1}(Z) x+\varepsilon$, where $\alpha_{1}(Z)=3, n=300, x$ is a normal random variable with a mean of 32 and standard deviation of $4, z$ is a random number from 0 to $8, \varepsilon$ is a normal random error with a mean of zero and standard deviation of 1 . This model has a constant slope. (b) Plot of $\hat{\alpha}_{1}(z)$ versus $z$ (dot) and $\alpha_{1}(Z)$ versus $z$ (line) for the data of (a), which shows the estimated $\hat{\alpha}_{1}(z)$ is approximated constant. (c) Plot of $y$ versus $x$ for the data simulated from the model $y=300+\alpha_{1}(Z) x+\varepsilon$, where $\alpha_{1}(Z)=0.5 z-2, n=300, x$ is a normal random variable with a mean of 100 and standard deviation of $12.5, z$ is a random number from 0 to $8, \varepsilon$ is a normal random error with a mean of zero and standard deviation of 0.2 . This model has a linear function of $\alpha_{1}(Z)$. (d) Plot of $\hat{\alpha}_{1}(z)$ versus $z$ (dot) and $\alpha_{1}(Z)$ versus $z$ (line) for the data in (b); which shows that the estimated $\hat{\alpha}_{1}(z)$ sufficiently approximates the real function $\alpha_{1}(Z)$.

one of the most well-studied interspecific cooperation systems. The fig provides some female flowers for the development of fig wasp offspring while fig wasps pollinate fig flowers, and therefore there is mutual benefit of the interaction $[41,42]$. In previous studies, the number of viable seeds of figs was used to measure the fitness of figs and the number of wasp offspring (galled flowers by fig wasps) was used to measure the fitness of fig wasps [43-45].

In the classical theories of the evolution of cooperation, including kin selection (Hamilton's rule), reciprocity selection (iterated prisoner's dilemma) or group selection, the Nash equilibrium or evolutionary stable strategy should exist between the cooperative actors [46]. The fitness correlation between cooperative actors should be uniquely positive $[11,12,32]$, and such a positive fitness correlation can be maintained by spatial heterogeneity or self-restraint of cooperative actors $[47,48]$. If there is a stable equilibrium in cooperative systems, a constant correlation should be expected between the fitness of the cooperative actors. In the fig-fig wasp mutualistic relationship, studies have found differences in whether or not the fitness correlation between fig and fig wasps is positively correlated $[43,45,49,50]$. Our analysis and experimental data showed that these conflicting findings on the fitness correlation result from problems associated with simple or multivariate linear regressions [27] because the fitness correlation coefficient greatly varies among different situations even for the same fig-fig wasp system $[27,31,44,45]$.

We employed the most commonly used method to analyze the correlation coefficient between viable seeds and wasp offspring to describe the relationship between figs and fig wasps. Data were randomly collected over different years from four sample sites to form a total number of samples of 225. The distributions of both the number of viable seeds and the number of wasp offspring were very noisy. However, such a noise might possibly be caused by chaos because it is difficult to distinguish between noise and chaos in this situation $[51,52]$.

We first examined whether the linear regression analysis could describe the relationship between the number of viable seeds and the number of wasp offspring, following graphical evaluation methods such as those of Sugihara and May [51]. Figure 3(a) and (b) clearly shows a general linear relationship for the simulated data of Figure 1(a). Figure 

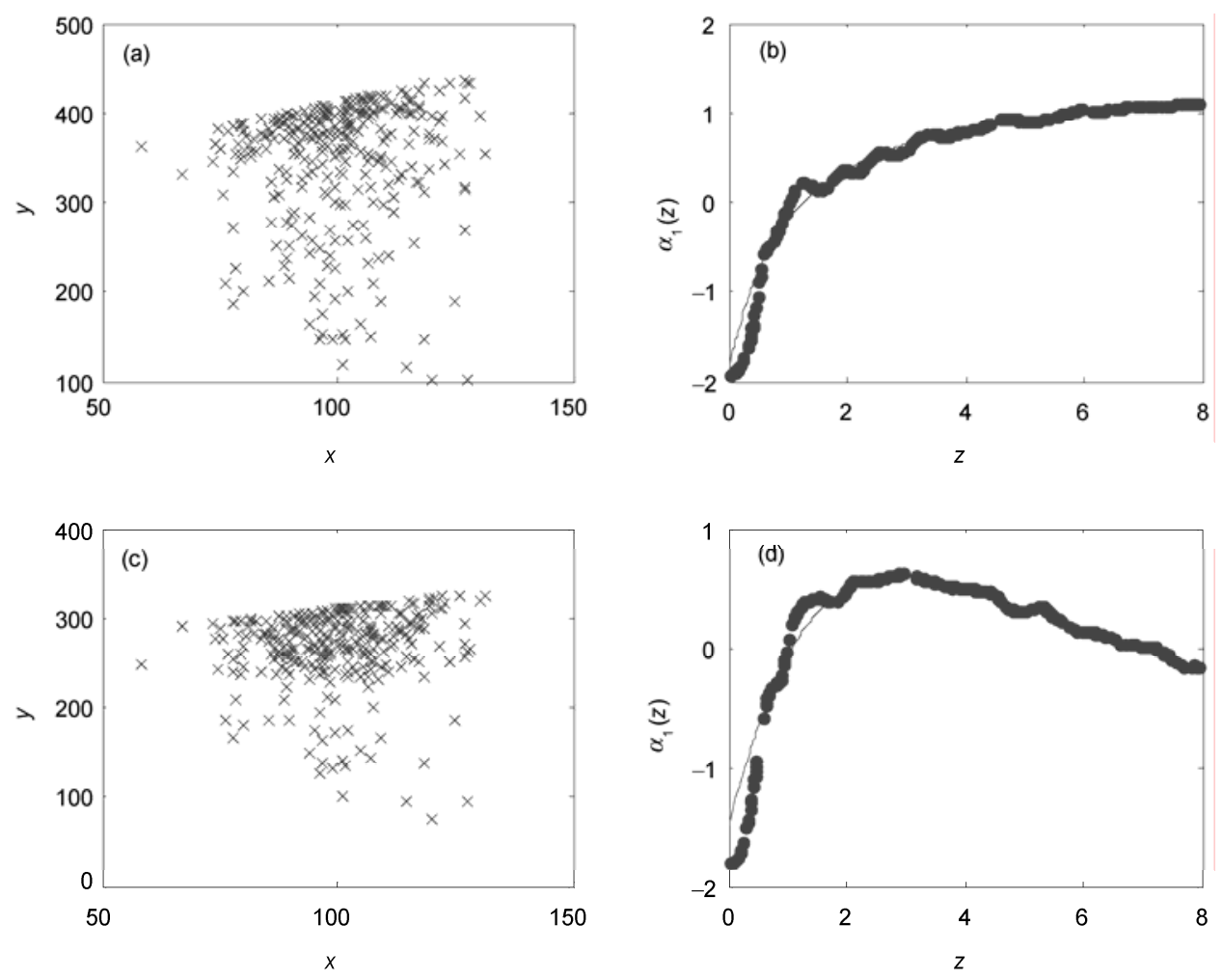

Figure 2 (a) Plot of $y$ versus $x$ for the data simulated from the model $y=300+\alpha_{1}(Z) x+\varepsilon$, where $\alpha_{1}(z)=\frac{1.5 z-1.9}{1+z}, n=300, x$ is a normal random variable with a mean of 100 and standard deviation of $12.5, z$ is a random number from 0 to $8, \varepsilon$ is a normal random error with a mean of zero and standard deviation of 0.2. (b) Plot of $\hat{\alpha}_{1}(z)$ versus $z$ (dot) and $\alpha_{1}(Z)$ versus $z$ (line) for the data of (a), which shows the estimated $\hat{\alpha}_{1}(z)$ sufficiently approximates $\alpha_{1}(Z)$. (c)

Plot of $y$ versus $x$ for the data simulated from the model $y=250+\alpha_{1}(Z) x+\varepsilon$, where $\alpha_{1}(z)=\frac{0.5+2 z}{1+0.2 z+0.1 z^{2}}-2, n=300, x, z$ and $\varepsilon$ are same as those in (a).

(d) Plot of $\hat{\alpha}_{1}(z)$ versus $z($ dot $)$ and $\alpha_{1}(Z)$ versus $z$ (line) for the data of (c), which shows that the estimated $\hat{\alpha}_{1}(z)$ sufficiently approximates the real function $\alpha_{1}(Z)$.

3(c) and (d) presents the real data for the fig-fig wasp mutualism (Ficus racemosa), showing that a constant linear regression is not appropriate when compared with the results in Figure 3(a) and (b). The $P$-value of the $F$-test for the linear regression model for the real data is 0.501 , and therefore obviously not significant.

Using the varying coefficient analysis developed in this paper, we show that the fitness correlation coefficient between figs and fig wasps varies with the fitness of fig wasps and the availability of resources (vacant female flowers) (Figure 4). This indicates that greater oviposition of foundresses can increase viable seed production when there are abundant vacant female flowers or when the number of oviposited flowers (galled flower) is small. This might be because pollen is dispersed to flowers during oviposition $[27,43]$, and therefore the number of viable seeds and wasp offspring can be simultaneously increased at the expense of unused flowers when there are many unused flowers. In this situation, a positive correlation between seed and wasp offspring production occurs. However, after most of the female flowers have been oviposited or pollinated, further oviposition is at the expense of viable seeds if flowers cannot prevent foundresses from using pollinated flowers or if foundresses cannot self-restrict their overexploitation of flowers. As previous quantitative measurements have shown that flower structure cannot sufficiently prevent foundresses from overexploiting flowers [27,52,53], a negative correlation between viable seed and wasp offspring production can therefore be observed when flowers have been all used or the number of oviposited flower is high.

The oviposition and pollination efficiency of foundresses, the number of foundresses and the female flower availability of figs can be expected to greatly vary among different situations, such as different habitats, seasons or even different crops, and therefore the correlation coefficient between viable seed and fig wasp offspring number might vary with any change of these factors [52]. This can fully explain why the fitness correlation differs for different availabilities of vacant female flowers [27], different resource allocations [44], and different seasons [31] or 

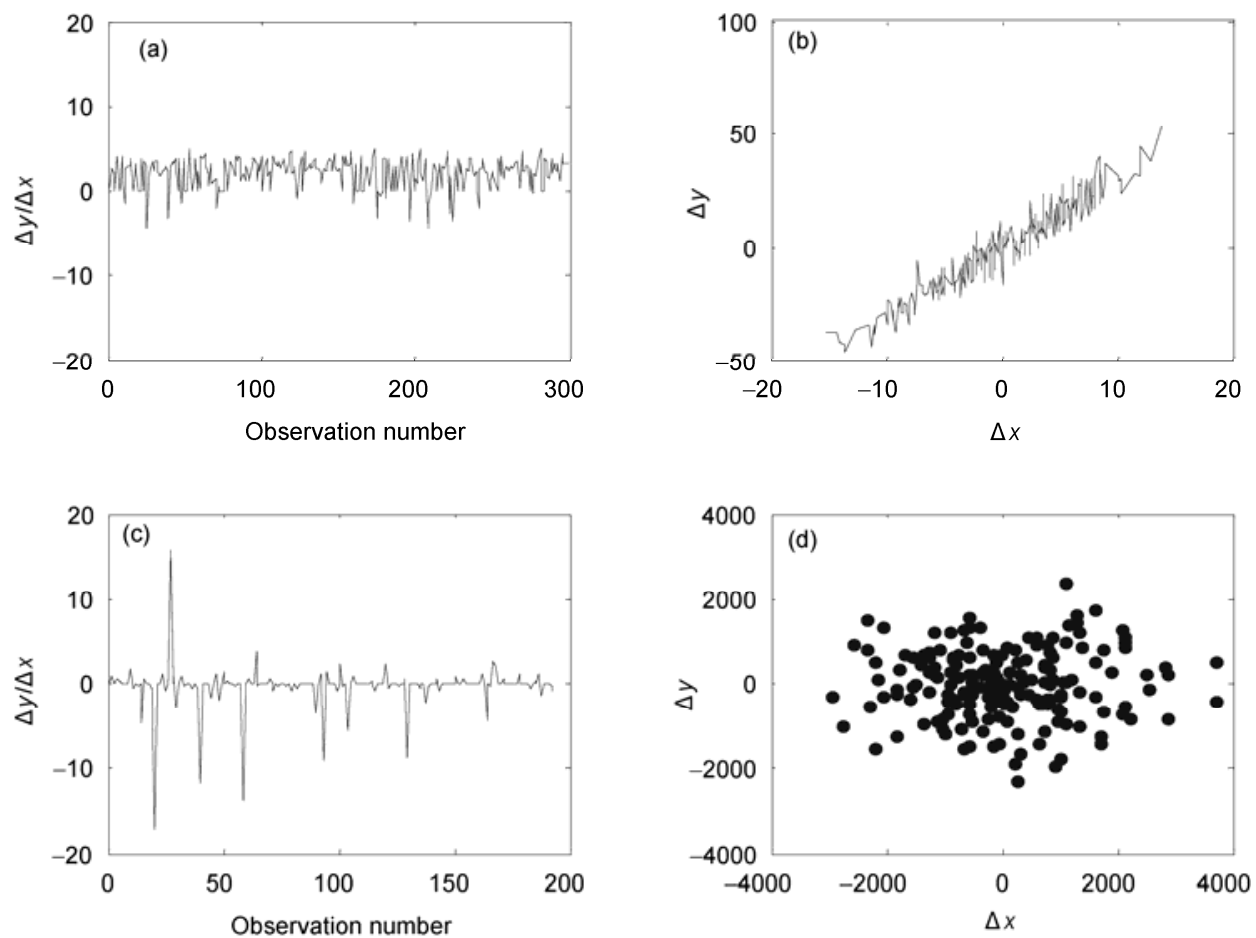

Figure 3 Plots for artificial data and real data of the fig-fig wasp mutualism. (a) Plot of $\Delta y / \Delta x$ versus observation number for the simulation data as shown in Figure 1(a), which indicates a constant linear regression of $y$ on $x$ is approproate since $\Delta y / \Delta x$ approximates as a constant. (b) Plot of $\Delta y$ versus $\Delta x$ for the simulation data, which shows a clear linear relationship. (c) Plot of $\Delta y / \Delta x$ versus observation number for the fig-fig wasp data, which is unstable and indicates that a constant linear regression of $y$ on $x$ is not appropriate. (d) Plot of $\Delta y$ versus $\Delta x$ for the fig-fig wasp data, which shows there is no clear linear relationship. $y=$ seeds, $x=$ wasp offspring (galls) in (c) and (d).
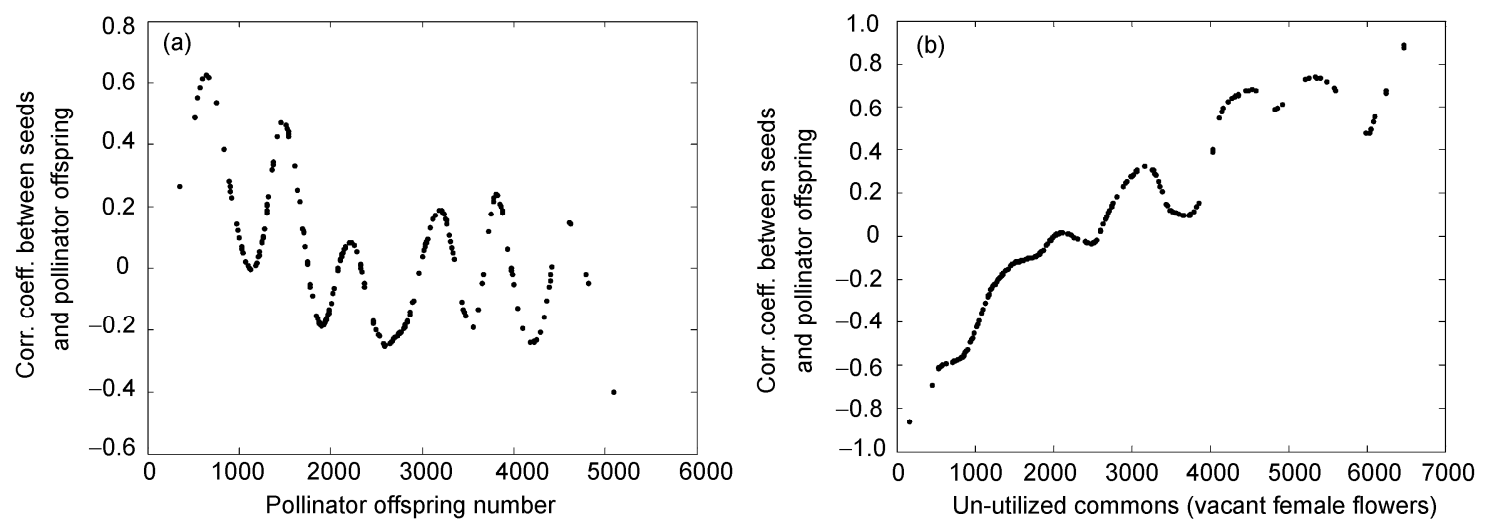

Figure 4 The correlation coefficient between the fitness of figs (viable seeds) and the fitness of pollinator wasps (offspring number) of the cooperating species interaction, using the varying correlation coefficient analysis. The figures indicate that the correlation coefficient oscillates as a function of wasp offspring in the fig-fig wasp reciprocal mutualism (a). There is a positive fitness correlation, when the common resource availability is high, while there is negative fitness correlation when the common resource availability is limited (b). For experimental evidence that the reciprocal mutualistic relationship depends on the common resource availability, see the paper of Wang et al. [27]. The smoothing curve of $\alpha_{0}(Z)$ where the kernel function is a quadratic kernel and the bandwidth $h$ is 500. The sample size was 225, and samples were collected over different years from 4 habitat sites.

different crops $[45,52]$. Our experimental data of the fig-fig wasp system showed that interference competition mainly existed among the fig wasps [36], and that asymmetric punishment of figs against non-cooperative fig wasps [54-56] leads to spatial heterogeneity and therefore an indeterminate fitness interaction. These results showed that the fitness interaction between cooperative players chaotically oscillated with either variation of the fitness density of the in- volved players or common resource availability, and that it is not a constant correlation as expected in the equilibrium theories of kin selection (Hamilton's rule), reciprocity selection (Iterated Prisoner's Dilemma) and group selection. Such a fitness correlation change has not found in the previous empirical studies partly because of the misuse of linear regression [27].

Nowak [57] has pointed out that that no mutant could 
invade equilibrium systems, and that a strict equilibrium or evolutionary stable strategy might be inaccessible in biological systems. Spatial chaos might be more possible, which has been theoretically argued in research on the evolution of cooperation [47,52,58,59], evolution of parasite-host or predator-prey relationships $[24,28]$ and population biology [60]. Using this varying coefficient method, the manner in which the species correlation varies with other factors can be sufficiently described in our data (Figure 4; Appendix A), providing direct evidence for changes in the fitness correlation.

Empirical data analysis results applying this method to coexisting and antagonistic species are available in the Appendix A.

\section{Discussion}

Both theoretical predictions and empirical data suggests that positive, negative and neutral species correlations can be observed in the same species interaction in cooperative (mutualism), antagonistic, and coexisting species systems [5,20,25-28,31]. The species correlation may greatly vary under different environmental or ecological conditions, or different fitnesses or population densities. In such cases, linear or non-linear regression (e.g. exponential or logistic) analysis cannot be employed to describe such a species interaction without a stable relationship. The varying coefficient analysis method, in which the functional responses of any specific quantitative characteristics of species are not assumed, is therefore appropriate. Through using this method, the manner in which the correlation coefficient of biological characteristics varies with other factors could be described in our empirical data analysis.

When the correlation coefficient is a specific value, the analysis for the interacting species using the varying coefficient regression model will reduce to a parametric analysis (Figure 1(a) and (b)). This means that this new method is more general and more credible than any existing parametric analysis when there is no prior information on the underlying model structure of the interacting species under investigation. Figures (Figure 1 (c) and (d); Figure 2 (a)(d)) of the simulated data showed that it is difficult to estimate the functional response from the data distribution and nonparametric estimation is necessary.

In the model, the effect of the third variable $Z$ can sometimes be integrated into the characteristics parameters of interacting species, and therefore we can use $X$ or $Y$ to replace $Z$ as the $x$-axis to describe how the correlation coefficient of interacting species varies according to changes of the characteristics of interacting species. Using $X$ or $Y$ to replace $Z$ will be simpler in practice in the following two cases: (1) The critical factors that determine the variation of the correlation coefficient of the interacting species cannot be easily identified, or only variables $X$ and $Y$ are available in practice; and (2) if the coefficient $\alpha_{1}$ of variable $Z$ is noisy or cannot be well described, using $X$ or $Y$ instead of $Z$ as the $x$-axis to integrate the effect of factor $Z$. Technical details for replacing $Z$ by $X$ or $Y$ is available in the Appendix B.

In traditional statistics, the effects of factors are usually considered as covariates. Such partial regression coefficient analysis can be used only when controlled factors are linear function of the interacting species [1,7,12,32]. Such a method can help to estimate the correlation coefficient of the interacting species. However, if the effect of these variables is not linear, the regression coefficient should be a function of other influential variables, and these variables cannot be regarded as covariates using parametric regression analysis. Simply including the influential variables as covariates using the parametric regression method only obtains an averaged value when these variables are controlled. This averaged value is unlikely to appropriately describe the true species interaction, and might even lead to an incorrect conclusion regarding the species relationship if these variables greatly affect the correlation coefficient [27].

We thank for Dr. Zhu LiPing and one anonymous reviewer for their useful comments and revisions suggested for this paper. This work was supported by the National Basic Research Program of China (2007CB411600), National Natural Science Foundation of China (30670272, 30770500 and 10761010), the Natural Science Foundation of Yunnan Province (2009CD104), the West Light Foundation of the Chinese Academy of Sciences, Special Fund for the Excellent Youth of the Chinese Academy of Sciences (KSCX2-EW-Q-9), State Key Laboratory of Genetic Resources and Evolution, the National Social Science Foundation of China (08XTJ001) and Research Grants Council of Hong Kong (HKBU2030/07P).

1 Li X C, Wang Z H, Wang W L. Biology Statistics (in Chinese). Beijing: Science Press, 2000

2 McCullagh P, Nelder J A. Generalized Linear Models (2nd ed). London: Chapman and Hall, 1989

3 Krebs J K. Ecology: The Experimental Analysis of Distribution and Abundance (2nd ed). New York: Harper and Row Press, 1978

4 May R M. Simple mathematical models with very complicated dynamics. Nature, 1976, 261: 459-467

5 Sun R Y. Fundamentals of Animal Ecology (in Chinese). Beijing: Beijing Normal University Press, 1992

6 Ma Z E. Theoretical Models of Population Ecology (in Chinese). Hefei: An-hui Education Press, 1996

7 Frank S A. The price equation. Fisher's fundamental theorem, kin selection and causal analysis. Evolution, 1997, 51: 1712-1729

8 Molles M C. Ecology: Concepts and Applications. New York: McGraw-Hill Companies, 1999

9 Whittingham M J, Stephen P A, Bradbury R B, et al. Why do we still use stepwise modeling in ecology and behaviour? J Anim Ecol, 2006, 75: 1182-1189

10 Fisher R A. The Genetical Theory of Natural Selection. Clarendon: Oxford, 1930

11 Price G R. Selection and covariance. Nature, 1970, 227: 520-521

12 Queller D C. A general model for kin selection. Evolution, 1992, 46: 376-380

13 Stiling P D. Ecology: Theories and Applications. New Jersey: Prentice Hall, Upper Saddle River, 1996

14 Draper N R, Smith H. Applied Regression Analysis. New York: John Wiley and Sons, 1981

15 Raubenheimer D. Problems with ratio analysis in nutritional studies. Functional Ecol, 1995, 9: 21-29

16 Inouye B D. Response of surface experiment design for investigating interspecific competition. Ecology, 2001, 82: 2696-2706

17 Travis J, Trexler J C. Interactions among factors affecting growth, 
development and survival in experimental populations of Bufo terrestris (Anura: Bufonidae). Oecologia (Berlin), 1986, 69: 110-116

18 Trexler J C, McCulloch C E, Travis J. How can the functional response best be determined? Oecologia, 1988, 76: 206-214

19 Holling C S. Resilience and stability of ecological systems. Ann Rev Ecol Syst, 1973, 4: 1-23

20 May R M. Theoretical Ecology: Principles and Applications. Oxford: Blackwell Scientific Publishers, 1981

21 Nowak A M, Bonhoeffer S, May R. Spatial games and the maintenance of cooperation. Proc Natl Acad Sci USA, 1994, 91: 4877-4881

22 Jiang L, Morin P J. Temperature fluctuation facilitates coexistence of competing species in experimental microbial communities. J Anim Ecol, 2007, 76: 660-668

23 Hofbauer J, Sigmund K. Evolutionary Games and Population Dynamics. Cambridge: Cambridge University Press, 1998

24 Hanski I, Turchin P, Korpimaki E, et al. Population oscillations of boreal rodents: Regulation by mustelid predators lead to chaos. Nature, 1993, 364: 232-235

25 Ives A R. Predicting the response of populations to environmental change. Ecology, 1995, 76: 926-941

26 Gonzalez A, Descamps-Julien B. Population and community variability in randomly fluctuating environments. Oikos, 2004, 106: 105-116

27 Wang R W, Shi L, Ai S M, et al. Trade-off between the reciprocal mutualists: Local resource availability oriented interaction in fig/fig wasp mutualism. J Anim Ecol, 2008, 77: 616-623

28 Hassell M P, Comins H N, May R M. Spatial structure and chaos in insect population dynamics. Nature, 1991, 353: 255-258

29 Thompson J N, Fernandez C C. Temporal dynamics of antagonism and mutualism in a geographically variable plant-insect interaction. Ecology, 2006, 87: 103-112

30 Smith J M, White A, Sherratt A J, et al. Disease effects on reproduction can cause population cycles in seasonal environments. J Anim Ecol, 2008, 77: 378-389

31 Wang R W, Yang J X, Yang D R. Seasonal changes in the trade-off among the fig-supported wasps and viable seeds in figs and their evolutionary implications. J Integr Plant Biol, 2005, 47: 144-155

32 Frank S A. Genetics of mutualism: The evolution of altruism between species. J Theor Biol, 1994, 170: 393-400

33 Sun B F, Wang R W, Hu Z, et al. The relation between two non-pollinating wasps oviposition and the fruit abscission on Ficus racemosa. Acta Ecol Sin, 2009, 29: 1-6

34 Rudolf V H W. Consequences of size structure in the prey for predator-prey dynamics: The composite functional response. J Anim Ecol, 2008, 77: 520-528

35 Wang R W, Sun B F. The seasonal change in the structure of fig-wasp community of figs and its implication for conservation. Symbiosis, 2009, 47: 77-83

36 Wang R W, Ridley J, Sun B F, et al. Interference competition and high temperatures reduce the virulence of fig wasps and stabilize a fig-wasp mutualism. PLoS ONE, 2009, 4: e7802

37 Hastie T, Tibshirani R. Varying-coefficient models (with discussion). J Roy Stati Soc B, 1993, 55: 757-796

38 Epanechnikov V. Nonparametric estimates of a multivariate probability density. Theory Probab Appl, 1969, 14: 153-158

39 Haerdle W. Applied Nonparametric Regression. Berlin: Springer,
1994

40 Zhu L X. Nonparametric Monte Carlo Tests and Their Applications. New York: Springer, 2005

41 Janzen D H. How to be a fig. Ann Rev Ecol Syst, 1979, 10: 13-51

42 Wiebes J T. Co-evolution of figs and their insect pollinators. Ann Rev Ecol Syst, 1979, 10: 1-12

43 Bronstein J L, Hossaert-McKey M. Variation in reproductive success within a subtropical fig-pollinator mutualism. J Evol Biol, 1996, 23: 433-446

44 Anstett M C, Bronstein J L, Hossaert-McKey M. Resource allocation: A conflict in the fig-fig wasp mutualism. J Evol Biol, 1996, 9: $417-428$

45 Herre E A, West S A. Conflict of interest in a mutualism: Documenting the elusive fig wasp-seed trade-off. Proc Roy Soc B, 1997, 264: 1501-1507

46 Maynard S J. Evolution and the Theory of Games. Cambridge: Cambridge University Press, 1982

47 Nowak A M, May M R. Evolutionary games and spatial chaos. Nature, 1992, 359: 826-829

48 Doebeli M, Knowlton N. The evolution of interspecific mutualisms. Proc Natl Acad Sci USA, 1998, 95: 8676-8680

49 Bronstein J L. Seed predator as mutualists: Ecology and evolution of the fig-pollinator interaction. In: Bernays E, ed. Insect-Plant Interaction. Boca Raton: CRC Press, 1992

50 Herre E A, Knowlton N, Mueller U G, et al. The evolution of mutualisms: Exploring the paths between conflict and cooperation. Trend Ecol Evol, 1999, 14: 49-53

51 Sugihara G, May R M. Non-linear forecasting as a way of distinguishing chaos from measurement error in time series. Nature, 1990, 344: 734-741

52 Wang R W, Sun B F, Zheng Q, et al. Asymmetric interaction and indeterminate fitness correlation between cooperative partners in the fig/fig wasp mutualism. J Roy Soc Interface, 2011, doi: 10.1098/rsif. 2011.0063

53 Nefdt R J C, Compton S G. Regulation of seed and pollinator production in the fig-fig wasp mutualism. J Anim Ecol, 1996, 65: $170-182$

54 Wang R W, Sun B F, Zheng Q. Diffusive co-evolution and mutualism maintenance mechanisms in a fig-fig wasp system. Ecology, 2010, 91: 1308-1316

55 Wang R W, Zheng Q. Structure of a figs wasp community: Temporal segregation of oviposition and larval diets. Symbiosis, 2008, 45: $113-116$

56 Wang R W, Sun B F. The seasonal change in the structure of fig-wasp community of figs and its implication for conservation. Symbiosis, 2009, 47: 77-83

57 Nowak A M. An evolutionary stable strategy may be inaccessible. J Theor Biol, 1990, 142: 237-241

58 Wang R W, Shi L. The evolution of cooperation in asymmetric systems. Sci China Life Sci, 2010, 53: 139-149

59 Wang R W, He J Z, Wang Y Q, et al. Asymmetric interaction will facilitate the evolution of cooperation. Sci China Life Sci, 2010, 52: 1041-1046

60 Tilman D, Wedin D. Oscillation and chaos in dynamics of a perennial grass. Nature, 1991, 353: 653-655

Open Access This article is distributed under the terms of the Creative Commons Attribution License which permits any use, distribution, and reproduction in any medium, provided the original author(s) and source are credited.

\section{Supporting Information}

Appendix A Empirical data of coexisting and antagonistic wasp species.

Appendix B An example of the factor $Z$.

The supporting information is available online at csb.scichina.com and www.springerlink.com. The supporting materials are published as submitted, without typesetting or editing. The responsibility for scientific accuracy and content remains entirely with the authors. 